\title{
Gravitational Waves from a Dark Phase Transition
}

\begin{abstract}
Pedro Schwaller
CERN, Theory Division, CH-1211 Geneva 23, Switzerland

(Received 1 June 2015; revised manuscript received 2 September 2015; published 26 October 2015)

In this work, we show that a large class of models with a composite dark sector undergo a strong first order phase transition in the early Universe, which could lead to a detectable gravitational wave signal. We summarize the basic conditions for a strong first order phase transition for $\mathrm{SU}(N)$ dark sectors with $n_{f}$ flavors, calculate the gravitational wave spectrum and show that, depending on the dark confinement scale, it can be detected at eLISA or in pulsar timing array experiments. The gravitational wave signal provides a unique test of the gravitational interactions of a dark sector, and we discuss the complementarity with conventional searches for new dark sectors. The discussion includes the twin Higgs and strongly interacting massive particle models as well as symmetric and asymmetric composite dark matter scenarios.
\end{abstract}

DOI: 10.1103/PhysRevLett.115.181101

PACS numbers: 04.30.-w, 12.60.-i, 95.35.+d

Introduction.-Violent phenomena in the early Universe can lead to large anisotropic fluctuations in the energy momentum tensor, which act as sources for gravitational waves $(\mathrm{GW})$. Strong first order phase transitions (PT) are examples of such a phenomena, and it is well known that they can produce GWs [1-4]. Once produced, GWs propagate through space almost undisturbed, and can therefore serve as a unique probe of processes in the early Universe.

Phase transitions in particle physics are usually associated with symmetry breaking, i.e., with the transition of the Universe from a symmetric phase to a phase of broken symmetry, as the temperature drops below a critical temperature $T_{*}$. Within the standard model (SM) of particle physics, at least two phase transitions should take place, associated with the breaking of electroweak symmetry (EWPT) around $T_{*} \sim 100 \mathrm{GeV}$ and with the breaking of chiral symmetry at the time of the QCD phase transition, $T_{*} \sim 0.1 \mathrm{GeV}$.

Today we know that both the QCD PT and the EWPT are not first order, but proceed through a smooth crossover [5-8], and can therefore not produce a strong GW signal through the usual mechanism. This can, however, be changed in models beyond the SM. Extensions of the SM which lead to a strong first order EWPT are particularly attractive since they can provide one missing ingredient for generating the observed baryon asymmetry of the Universe [9-15]. It is more difficult to modify the QCD PT, although a large neutrino chemical potential could be sufficient to provide a strong first order PT [16]. The resulting signal was studied in Ref. [17].

The aim of this work is to point out that gravitational waves could also be produced by a strong PT in a dark or hidden

Published by the American Physical Society under the terms of the Creative Commons Attribution 3.0 License. Further distribution of this work must maintain attribution to the author(s) and the published article's title, journal citation, and DOI. sector. The particular scenario we have in mind is a dark sector with a new $\mathrm{SU}\left(N_{d}\right)$ gauge interaction which confines at some scale $\Lambda_{d}$. Such models have recently received renewed interest either as models of dark matter [18-32] or as part of the low energy sector of so-called twin Higgs models [33-35]. Different from generic hidden sectors [36], these models provide a preferred mass range and some restrictions on the particle content, such that the frequency range of the potential GW signal can be predicted.

Models with first order phase transition.-Near the confinement scale $\Lambda_{\mathrm{QCD}}$, the dynamics of QCD is governed by three flavors, two of which are almost massless, while the strange quark mass is of order $\Lambda_{\mathrm{QCD}}$. Lattice studies $[5,6,37]$ have shown that for these quark masses, the QCD PT is a weak crossover. This is, however, not always the case. For different values of $m_{u, d}$ and $m_{s}$ the behavior is as follows: The pure Yang-Mills limit $m_{u, d}, m_{s} \rightarrow \infty$ is known to have a strong first order PT [38] from the restoration of a global $Z_{3}$ center symmetry at low temperatures. The opposite $m_{u, d}, m_{s} \rightarrow 0$ limit, i.e., the theory with three exactly massless quarks, also features a strong first order transition, related to the breakdown of the $\mathrm{SU}(3) \times \mathrm{SU}(3)$ chiral symmetry [39].

Here we are interested in $\mathrm{SU}\left(N_{d}\right)$ theories with $N_{d} \geq 3$ and $n_{f}$ massless $\left(m \ll \Lambda_{d}\right)$ Dirac fermions in the fundamental representation, and with a confinement scale $\Lambda_{d}$. To guarantee the existence of a confining phase we further impose $n_{f}<4 N_{d}$, in order to stay outside of the conformal window.

For pure Yang-Mills theories, $n_{f}=0$, the confinement phase transition is related to the restoration of a global $Z_{N_{d}} \subset \mathrm{SU}\left(N_{d}\right)$ center symmetry, which is broken in the high temperature phase. Lattice simulations have shown that this PT is strong first order for $N_{d} \geq 3$ [40].

The case of nonzero $n_{f}$ for $N_{d}=3$ and $N_{d} \rightarrow \infty$ is discussed in Ref. [39]. For $n_{f}=1$ there is no symmetry breaking, and therefore no phase transition. For $n_{f} \geq 3$ the 
PT is first order for any value of $N_{d} \geq 3$. In the large $N_{d}$ limit, the PT is also first order for $n_{f}=2$.

To summarize, $\mathrm{SU}\left(N_{d} \geq 3\right)$ theories with $n_{f}$ massless flavors have a first order PT if either $n_{f}=0$ or $3 \leq n_{f}<4 N_{d}$. In the following, we will discuss a few examples that are well motivated either from a dark matter perspective or by naturalness arguments. These physically motivated scenarios furthermore provide a preferred range for the confinement scale $\Lambda_{d}$, which allows us to estimate the temperature of the PT, $T_{*} \sim \Lambda_{d}$, and therefore make a prediction for the $\mathrm{GW}$ spectrum.

Composite dark matter I (CDM1).-In this class of models, the dark matter candidate is the lightest baryonic bound state of a $\mathrm{SU}\left(N_{d}\right)$ dark sector with $n_{f}$ dark quarks which are neutral with respect to all SM interactions. This allows the DM to be light, since it will only communicate with the SM through heavy mediators. The most natural realization of these models is in the context of asymmetric dark matter [41,42]. The measured DM density then implies a mass range of $\mathcal{O}(5) \mathrm{GeV}$ for the DM particle, and therefore motivates $T_{*} \approx \Lambda_{d} \sim(1-10) \mathrm{GeV}$. As a concrete benchmark we will consider the dark QCD model [24,31], which consists of a $\mathrm{SU}\left(N_{d}=3\right)$ theory with $n_{f} \geq$ 3 dark quarks, such that a strong PT is guaranteed.

Composite dark matter II (CDM2).--Similar to the previous case, the DM is a baryonic bound state of a new $\mathrm{SU}\left(N_{d}\right)$ theory. The important difference now is that the dark quarks carry electroweak quantum numbers. Models of this type were considered, for example, in Refs. [21,22], where mass spectra and form factors are calculated from first principles on the lattice. Constraints on the invisible width of the $Z$ boson immediately suggest a confinement scale at or above the $100 \mathrm{GeV}$ scale, while unitarity arguments on DM freeze-out place a limit of $\Lambda_{d} \lesssim \mathcal{O}(100) \mathrm{TeV}$. The benchmark model considered in Ref. [22] has $N_{d}=4$ with $n_{f}=4$ flavors and should therefore feature a strong first order PT.

Twin Higgs (TH).- These models attempt to solve the hierarchy problem without introducing coloured partners for the top quark $[33,34]$. This is achieved by adding a twin sector to the SM with an approximate $Z_{2}$ parity symmetry, with the minimal requirement that there should be fermionic partners for the top and bottom quarks which are charged under a new $\mathrm{SU}\left(N_{d}=3\right)$ interaction. The approximate $Z_{2}$ symmetry constrains $\Lambda_{d}$ to lie in the (1-10) GeV range. Top and bottom partners are heavier such that the theory at the PT scale is $\mathrm{SU}(3)$ with $n_{f}=0$ [35], which again gives a strong first order PT.

Strongly interacting massive particle (SIMP).-The key idea behind SIMP DM is that the relic density is determined through freeze-out of $3 \rightarrow 2$ instead of $2 \rightarrow 2$ annihilations [43]. The correct relic abundance is then obtained for DM masses in the $100 \mathrm{MeV}$ range. One simple realization of this mechanism is a $\mathrm{SU}\left(N_{d}\right)$ dark sector with dark pions as DM candidates. The $3 \rightarrow 2$ processes can then be induced by the Wess-Zumino-Witten term $[44,45]$. The condition for the existence of the WZW term is $n_{f} \geq 3$, which again allows for a strong first order PT, this time in the $100 \mathrm{MeV}-\mathrm{GeV}$ range.

Gravitational wave spectra.-Gravitational waves produced at a time $t_{*}$ (or, equivalently, at a temperature $T_{*}$ ) will propagate undisturbed in the expanding Universe; therefore, their frequency $f$ and their fraction of the critical energy density $\Omega_{\mathrm{GW}}$ will decrease as $a^{-1}$ and $a^{-4}$, respectively, where $a(t)$ is the scale factor $[9,46]$. Denoting by $a_{*}$ and $a_{0}$, the scale factors at time of production and today, entropy conservation $\left(s a^{3}=\right.$ const $)$ implies

$$
\frac{a_{*}}{a_{0}}=\left(\frac{g_{0, s}}{g_{*, s}}\right)^{\frac{1}{3}} \frac{T_{0}}{T_{*}} .
$$

Here, $T_{0}=2.725 \mathrm{~K}=2.348 \times 10^{-13} \mathrm{GeV}$ is the temperature of the CMB, $g_{*, s}\left(g_{0, s}=3.91\right)$ is the effective number of relativistic degrees of freedom at the time of production (today), and the entropy density at temperature $T$ is given by $s(T)=\left[(2 \pi)^{2} / 45\right] g_{s}(T) T^{3}$. It follows that the frequency today can be expressed as

$$
f=\frac{a_{*}}{a_{0}} f_{*}=1.6 \times 10^{-7} \mathrm{~Hz} \times\left(\frac{g_{*}}{80}\right)^{\frac{1}{6}} \frac{T_{*}}{1 \mathrm{GeV}} \frac{f_{*}}{H_{*}},
$$

where we have used the Hubble rate at time of production, $H_{*}=\sqrt{\left(4 \pi^{3} g_{*} / 45\right)}\left(T_{*}^{2} / M_{\mathrm{Pl}}\right)$, and assumed that all species are in thermal equilibrium at $T=T_{*}$, i.e., $g_{*}=g_{*, s}$.

To understand the spectrum of GWs from a strong dark PT, we follow the discussion of Ref. [17]. Gravitational waves are sourced by tensor fluctuations of the energy momentum tensor of the primordial plasma. During first order PTs both bubble collisions [47-49] and magnetohydrodynamical (MHD) turbulence [50-56] provide sources of GWs. As functions of the conformal wave number $k=2 \pi a f$, the GW spectra produced by either source can be approximated by [17,57-59]

$$
\begin{aligned}
\frac{d \Omega_{\mathrm{GW}}^{(\mathrm{B})} h^{2}}{d \log k} & \simeq \frac{2}{3 \pi} h^{2} \Omega_{r 0}\left(\frac{\mathcal{H}_{*}}{\beta}\right)^{2} \Omega_{S *}^{2} v^{3} \frac{(k / \beta)^{3}}{1+(k / \beta)^{4}}, \\
\frac{d \Omega_{\mathrm{GW}}^{(\mathrm{MHD})} h^{2}}{d \log k} \simeq & \frac{8}{\pi^{6}} h^{2} \Omega_{r 0}\left(\frac{\mathcal{H}_{*}}{\beta}\right) \Omega_{S *}^{3 / 2} v^{4} \\
& \times \frac{(k / \beta)^{3}}{\left(1+4 k / \mathcal{H}_{*}\right)\left[1+\left(v / \pi^{2}\right)(k / \beta)\right]^{11 / 3}} .
\end{aligned}
$$

Here $\mathcal{H}_{*}$ is the conformal Hubble parameter $\mathcal{H}=\mathrm{Ha}$ at $T=T_{*}$, and $\Omega_{r 0}$ is the radiation energy density today. The quantities that determine the GW spectrum are the duration of the PT $\beta^{-1}$ [usually $(1-100) \%$ of a Hubble time [2]], the bubble velocity $v$, and the relative energy density in the 


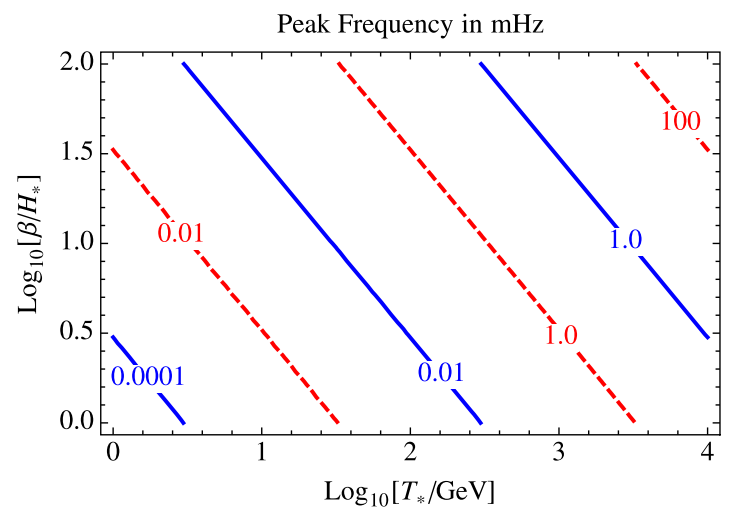

FIG. 1 (color online). Peak frequencies of the GW spectra (in $\mathrm{mHz}$ ) from bubble collisions (blue,solid) and MHD turbulence (red, dashed) in the $T_{*}-\beta$ plane, for $v=1.0$.

source, $\Omega_{S *}=\rho_{S *} / \rho_{* \text {, cirt }}$. The temperature of the PT enters through the dependence of $\mathcal{H}_{*}$ on $T_{*}$.

The spectra depend on the wave number only through $k / \mathcal{H}_{*}$ (since $\beta \propto \mathcal{H}_{*}$ ). Using Eq. (2) and $f_{*} / H_{*}=$ $\left(k / \mathcal{H}_{*}\right) /(2 \pi)$, we find the peak frequencies today,

$$
\begin{aligned}
f_{\text {peak }}^{(\mathrm{B})} & =3.3 \times 10^{-8} \mathrm{~Hz} \times\left(\frac{g_{*}}{80}\right)^{\frac{1}{6}}\left(\frac{T_{*}}{1 \mathrm{GeV}}\right)\left(\frac{\beta}{\mathcal{H}_{*}}\right), \\
f_{\text {peak }}^{(\mathrm{MHD})} & \approx 10 f_{\text {peak }}^{(\mathrm{B})},
\end{aligned}
$$

for $v \lesssim 1$. In Fig. 1 we show the location of the frequency peaks as functions of $T_{*}$ and $\beta$. As expected from Eq. (2), the peak frequencies increase linearly with the transition temperature $T_{*}$ and with $\beta / \mathcal{H}_{*}$.

Obviously, the signal strength strongly depends on the parameters $\beta, v$, and $\Omega_{S *}$, which are not known for the models considered here. In Ref. [60] it was shown that the radion-stabilized RS model, which is a dual description for strongly coupled gauge theories, has a very strong first order PT. For very strong PTs the bubble wall velocity approaches $v=1$ and large supercooling can provide $\Omega_{S *} \lesssim 1$. Furthermore, it was found that $\beta / \mathcal{H}_{*} \leq 10$ is possible, which gives rise to a larger signal than in weakly coupled models, where typically $\beta / \mathcal{H}_{*} \sim 10-100$ [61].

Motivated by the results of Ref. [60] we consider the GW signal for $v=1, \beta / \mathcal{H}_{*}=1-100$, and $\Omega_{S *}=0.1$ in the following (a similar range of values was also chosen in Ref. [17]).

Lattice simulations of the strongly coupled transitions will be necessary to determine $\beta, v$, and $\Omega_{S *}$ for different $N_{d}$ and $n_{f}$. In particular, an accurate determination of $\beta$ would be important since it determines both the peak location and the intensity of the signal. Finally, it should be noted that investigations of the GW spectrum from PTs is still ongoing (e.g., Refs. [62-64]), and that not all aspects of the MHD turbulence signal are captured by Eq. (4).

Detectability and complementarity.-We have seen that the peak frequencies of $\mathrm{GW}$ signals from $\mathrm{GeV}-\mathrm{TeV}$ scale PTs are of order $\left(10^{-6}-10^{-3}\right)$ Hz. GWs with frequencies down to $10^{-5} \mathrm{~Hz}$ can be probed by satellite based experiments like eLISA [65]; however, the sensitivity quickly degrades below $10^{-3} \mathrm{~Hz}$. On the other end of the spectrum, pulsar timing arrays (PTA) can probe frequencies in the $\left(10^{-9}-10^{-7}\right) \mathrm{Hz}$ range. In Fig. 2 we overlay the expected GW signal for different model parameters with the expected sensitivities of current and planned GW detection experiments (based on Ref. [66]).

PTA experiments [67-69] are very sensitive to the GW signal from models with a PT at the GeV scale or below,

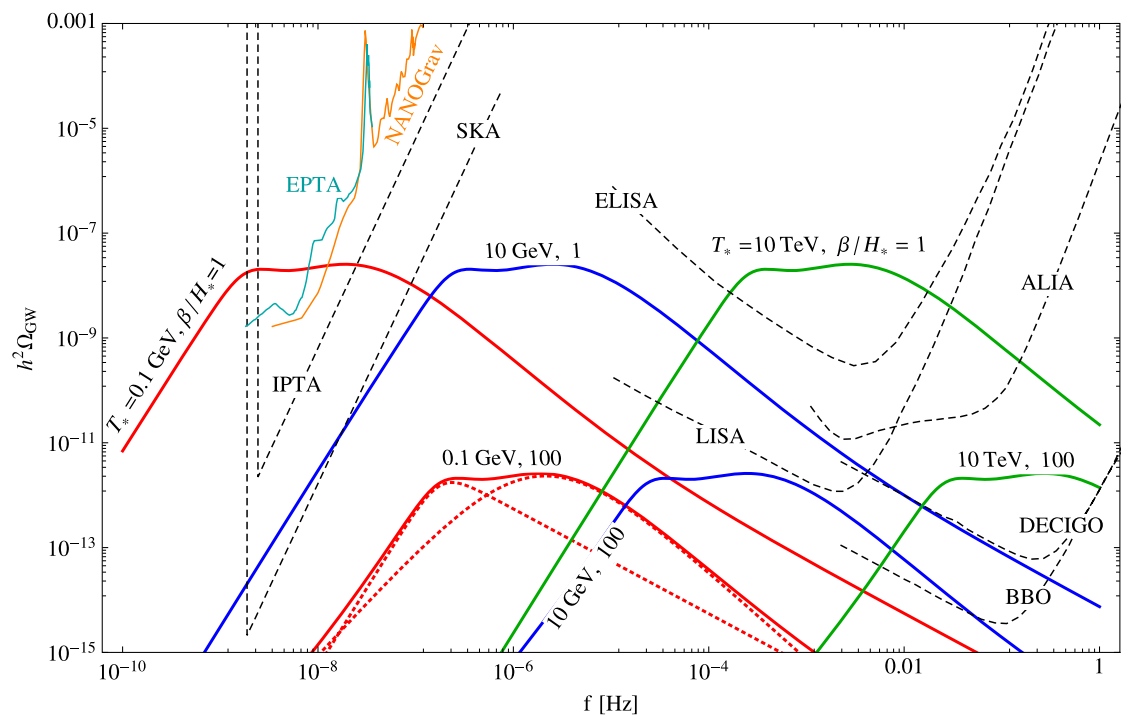

FIG. 2 (color online). $\quad$ GW spectra $\Omega(f) h^{2}$ for $T_{*}=0.1 \mathrm{GeV}$ (SIMP), $T_{*}=10 \mathrm{GeV}\left(\mathrm{CDM} 1, \mathrm{TH}\right.$ models) and $T_{*}=10 \mathrm{TeV}(\mathrm{CDM} 2$ models). Curves are shown for $\beta / \mathcal{H}_{*}=1,100, v=1$, and $\Omega_{S *}=0.1$. Dotted lines indicate the individual contributions from bubble collisions and turbulence. The projected reach of several planned GW detection experiments is shown (dashed lines). Limits on a stochastic GW background from the EPTA [67] and NANOGrav [68] experiments are also shown. 
which includes SIMP DM but also the QCD PT if it were strong first order. As the transition temperature increases the signals quickly fall out of the sensitivity range of PTAs, such that the CDM1 and TH models are only detectable here if their PT has $\beta \approx \mathcal{H}$. Recently, the EPTA [67] and NANOGrav [68] Collaborations have reported limits on the stochastic GW background as indicated in Fig. 2. It is interesting to note that GWs from a $100 \mathrm{MeV}$ scale dark sector could soon be detectable, although a precise estimate of the sensitivity would require determining the signal-tonoise ratio for the particular shape of the GW signal.

Satellite based experiments like eLISA and BBO are instead highly sensitive to models with PTs in the TeV range. Models of the CDM2 type naturally fall into this region, but also the CDM1 models can be viable with a confinement scale in that region. Another interesting aspect of these experiments is that they are sensitive to the tails of signals that peak at lower frequency, like the $T_{*}=10 \mathrm{GeV}$ band in Fig. 2. An interesting feature here is that the frequencies shift to larger values with $(\beta / \mathcal{H})$, which partially compensates the overall $(\mathcal{H} / \beta)^{2}$ drop of the signal in the high frequency tails. Therefore, satellite based experiments have the potential to probe a large range of PT temperatures from few $\mathrm{GeV}$ to $100 \mathrm{~s}$ of $\mathrm{TeV}$, although the distinction from other $\mathrm{GW}$ sources (for example, unresolvable galactic white dwarf binaries, contribute to the stochastic background at the level of $h^{2} \Omega_{\mathrm{GW}} \sim 10^{-10}$ in the $f=10^{-4}-10^{-3}$ range) could be difficult if the peak is not in the sensitivity range.

A distinct feature of GW signals is that it directly probes the gravitational effects of new physics sectors, whereas dark matter direct detection or collider experiments have to rely on sufficiently strong nongravitational interactions of the dark sectors through mediator particles. All of the models introduced earlier remain viable even if the masses or couplings of the mediators are adjusted such that their detection becomes difficult in current collider and DM detection experiments. The nonobservation of new physics in the near future would therefore not exclude the possibility of observing a GW signal from a dark sector. This is very different from, e.g., models of strong EWPT $[60,70,71]$, which could be in trouble if no new physics is discovered at the LHC. A GW signal in the absence of new physics discoveries would therefore point towards a dark sector, assuming it could be disentangled from astrophysical foregrounds.

Finally, it is worth noting that perturbative unitarity constrains the mass of thermal DM to be below $110 \mathrm{TeV}$ [72,73], beyond the reach of the next generation of collider experiments. For composite nonperturbative DM this limit does not apply directly, instead, a lower bound on the radius of the extended object can be obtained, $R \lesssim(100 \mathrm{TeV})^{-1}$. It is reasonable to expect the radius $R$ to be of order of the inverse mass, which again implies an upper bound on the DM mass of order
$100 \mathrm{TeV}$. GW signals could therefore be a unique probe of the thermal DM paradigm.

Conclusions.-Models beyond the SM with a confining dark sector can lead to unexpected phenomenological signatures. Here we have explored the possibility to detect gravitational waves due to a first order phase transition at the confinement scale $\Lambda_{d}$. DM and/or naturalness considerations constrain the scale of the phase transition, and the resulting GW frequencies lie in the sensitivity range of current or planned GW detection experiments. Depending on other aspects of the model, GW signals will either provide complementary information about the models in question, or might even be the best option to find evidence for these models of new physics.

It will be interesting to further study the PT in strongly coupled systems, to obtain a more precise understanding of the GW spectrum, and its dependence on the number of colors and flavors in the dark sector. Progress can be made both using lattice simulations and using holographic methods, opening a new connection between studies of strongly coupled $\mathrm{SU}(N)$ theories and cosmology.

I would like to thank M. Laine for very valuable discussions regarding the order of the PT and for encouragement and comments on the manuscript. Furthermore, I would like to thank T. Cohen, A. Kurkela, T. Konstandin, M. Mccullough, A. Petiteau, D. Stolarski, and A. Weiler for useful discussions and comments on the manuscript, and the organizers and participants of the eLISA and Neutral Naturalness workshops at CERN for providing a stimulating environment to finalize this work.

[1] E. Witten, Phys. Rev. D 30, 272 (1984).

[2] C. J. Hogan, Phys. Lett. B 133, 172 (1983).

[3] C. J. Hogan, Mon. Not. R. Astron. Soc. 218, 629 (1986).

[4] M. S. Turner and F. Wilczek, Phys. Rev. Lett. 65, 3080 (1990).

[5] Y. Aoki, G. Endrodi, Z. Fodor, S. D. Katz, and K. K. Szabo, Nature (London) 443, 675 (2006).

[6] T. Bhattacharya, M. I. Buchoff, N. H. Christ, H.-T. Ding, R. Gupta, C. Jung, F. Karsch, and Z. Lin et al., Phys. Rev. Lett. 113, 082001 (2014).

[7] K. Kajantie, M. Laine, K. Rummukainen, and M. E. Shaposhnikov, Nucl. Phys. B466, 189 (1996).

[8] K. Kajantie, M. Laine, K. Rummukainen, and M. E. Shaposhnikov, Phys. Rev. Lett. 77, 2887 (1996).

[9] C. Grojean and G. Servant, Phys. Rev. D 75, 043507 (2007).

[10] C. Delaunay, C. Grojean, and J. D. Wells, J. High Energy Phys. 04 (2008) 029.

[11] S. J. Huber and T. Konstandin, J. Cosmol. Astropart. Phys. 05 (2008) 017.

[12] S. Das, P. J. Fox, A. Kumar, and N. Weiner, J. High Energy Phys. 11 (2010) 108.

[13] J. R. Espinosa, T. Konstandin, J. M. No, and M. Quiros, Phys. Rev. D 78, 123528 (2008). 
[14] M. Jarvinen, C. Kouvaris, and F. Sannino, Phys. Rev. D 81, 064027 (2010).

[15] J. R. Espinosa, T. Konstandin, J. M. No, and G. Servant, J. Cosmol. Astropart. Phys. 06 (2010) 028.

[16] D. J. Schwarz and M. Stuke, J. Cosmol. Astropart. Phys. 11 (2009) 025; 10 (2010) E01.

[17] C. Caprini, R. Durrer, and X. Siemens, Phys. Rev. D 82, 063511 (2010).

[18] M. R. Buckley and E. T. Neil, Phys. Rev. D 87, 043510 (2013).

[19] T. Hambye and A. Strumia, Phys. Rev. D 88, 055022 (2013).

[20] G. C. Dorsch, S. J. Huber, and J. M. No, Phys. Rev. Lett. 113, 121801 (2014).

[21] T. Appelquist et al. (Lattice Strong Dynamics (LSD) Collaboration), Phys. Rev. D 88, 014502 (2013).

[22] T. Appelquist, R. C. Brower, M. I. Buchoff, G. T. Fleming, X. Y. Jin, J. Kiskis, G. D. Kribs, E. T. Neil et al., Phys. Rev. D 92, 075030 (2015).

[23] O. Antipin, M. Redi, A. Strumia, and E. Vigiani, J. High Energy Phys. 07 (2015) 039.

[24] Y. Bai and P. Schwaller, Phys. Rev. D 89, 063522 (2014).

[25] S. Bhattacharya, B. Melić, and J. Wudka, J. High Energy Phys. 02 (2014) 115.

[26] J. M. Cline, Z. Liu, G. Moore, and W. Xue, Phys. Rev. D 90, 015023 (2014).

[27] K. K. Boddy, J. L. Feng, M. Kaplinghat, and T. M. P. Tait, Phys. Rev. D 89, 115017 (2014).

[28] J. L. Newstead and R. H. TerBeek, Phys. Rev. D 90, 074008 (2014).

[29] G. Krnjaic and K. Sigurdson, arXiv:1406.1171.

[30] W. Detmold, M. McCullough, and A. Pochinsky, Phys. Rev. D 90, 115013 (2014).

[31] P. Schwaller, D. Stolarski, and A. Weiler, J. High Energy Phys. 05 (2015) 059.

[32] T. Cohen, M. Lisanti, and H. K. Lou, Phys. Rev. Lett. 115, 171804 (2015).

[33] Z. Chacko, H. S. Goh, and R. Harnik, Phys. Rev. Lett. 96, 231802 (2006).

[34] Z. Chacko, H. S. Goh, and R. Harnik, J. High Energy Phys. 01 (2006) 108.

[35] N. Craig, A. Katz, M. Strassler, and R. Sundrum, J. High Energy Phys. 07 (2015) 105.

[36] M. J. Strassler and K. M. Zurek, Phys. Lett. B 651, 374 (2007).

[37] Y. Aoki, Z. Fodor, S. D. Katz, and K. K. Szabo, Phys. Lett. B 643, 46 (2006).

[38] B. Svetitsky and L. G. Yaffe, Nucl. Phys. B210, 423 (1982).

[39] R. D. Pisarski and F. Wilczek, Phys. Rev. D 29, 338 (1984).

[40] M. Panero, Phys. Rev. Lett. 103, 232001 (2009).

[41] K. Petraki and R. R. Volkas, Int. J. Mod. Phys. A 28, 1330028 (2013).

[42] K. M. Zurek, Phys. Rep. 537, 91 (2014).

[43] Y. Hochberg, E. Kuflik, T. Volansky, and J. G. Wacker, Phys. Rev. Lett. 113, 171301 (2014).
[44] Y. Hochberg, E. Kuflik, H. Murayama, T. Volansky, and J. G. Wacker, Phys. Rev. Lett. 115, 021301 (2015).

[45] H. M. Lee and M. S. Seo, Phys. Lett. B 748, 316 (2015).

[46] M. Kamionkowski, A. Kosowsky, and M. S. Turner, Phys. Rev. D 49, 2837 (1994).

[47] A. Kosowsky, M. S. Turner, and R. Watkins, Phys. Rev. D 45, 4514 (1992).

[48] A. Kosowsky and M. S. Turner, Phys. Rev. D 47, 4372 (1993).

[49] C. Caprini, R. Durrer, and G. Servant, Phys. Rev. D 77, 124015 (2008).

[50] C. J. Hogan, Phys. Rev. Lett. 51, 1488 (1983).

[51] A. Kosowsky, A. Mack, and T. Kahniashvili, Phys. Rev. D 66, 024030 (2002).

[52] A. D. Dolgov, D. Grasso, and A. Nicolis, Phys. Rev. D 66, 103505 (2002).

[53] C. Caprini and R. Durrer, Phys. Rev. D 74, 063521 (2006).

[54] G. Gogoberidze, T. Kahniashvili, and A. Kosowsky, Phys. Rev. D 76, 083002 (2007).

[55] T. Kahniashvili, A. Kosowsky, G. Gogoberidze, and Y. Maravin, Phys. Rev. D 78, 043003 (2008).

[56] T. Kahniashvili, L. Kisslinger, and T. Stevens, Phys. Rev. D 81, 023004 (2010).

[57] C. Caprini, R. Durrer, T. Konstandin, and G. Servant, Phys. Rev. D 79, 083519 (2009).

[58] S. J. Huber and T. Konstandin, J. Cosmol. Astropart. Phys. 09 (2008) 022.

[59] C. Caprini, R. Durrer, and G. Servant, J. Cosmol. Astropart. Phys. 12 (2009) 024.

[60] T. Konstandin, G. Nardini, and M. Quiros, Phys. Rev. D 82, 083513 (2010).

[61] J. T. Giblin and E. Thrane, Phys. Rev. D 90, 107502 (2014).

[62] M. Hindmarsh, S. J. Huber, K. Rummukainen, and D. J. Weir, Phys. Rev. Lett. 112, 041301 (2014).

[63] M. Hindmarsh, S. J. Huber, K. Rummukainen, and D. J. Weir, arXiv:1504.03291.

[64] T. Kalaydzhyan and E. Shuryak, Phys. Rev. D 91, 083502 (2015).

[65] P. A. Seoane et al. (eLISA Collaboration), arXiv:1305.5720.

[66] C. J. Moore, R. H. Cole, and C. P. L. Berry, Classical Quantum Gravity 32, 015014 (2015).

[67] L. Lentati, S. R. Taylor, C. M. F. Mingarelli, A. Sesana, S. A. Sanidas, A. Vecchio, R. N. Caballero, and K. J. Lee et al., Mon. Not. R. Astron. Soc. 453, 2576 (2015).

[68] Z. Arzoumanian et al. (NANOGrav Collaboration), arXiv:1508.03024.

[69] R. N. Manchester et al., Pub. Astron. Soc. Aust. 30, e017 (2013).

[70] L. Randall and G. Servant, J. High Energy Phys. 05 (2007) 054

[71] T. Konstandin and G. Servant, J. Cosmol. Astropart. Phys. 12 (2011) 009.

[72] K. Griest and M. Kamionkowski, Phys. Rev. Lett. 64, 615 (1990).

[73] K. Blum, Y. Cui, and M. Kamionkowski, Phys. Rev. D 92, 023528 (2015). 